\title{
ArcheoSciences
}

Revue d'archéométrie

$34 \mid 2010$

Varia

\section{Mines d'argent du Montaigu (Hautes-Pyrénées, France)}

Une filière aquitaine de l'argent ? Étude isotopique du plomb

Silver mines on the massif of Montaigu (Hautes-Pyrénées, France): An

Aquitanian network for silver? Study on lead isotopes

Jérôme Girard, Marguerite Munoz, Béatrice Cauuet, Mireille Polve, Sébastien Aries et Laurent Callegarin

\section{OpenEdition}

\section{Journals}

Édition électronique

URL : https://journals.openedition.org/archeosciences/2792

DOI : $10.4000 /$ archeosciences. 2792

ISSN : 2104-3728

Éditeur

Presses universitaires de Rennes

Édition imprimée

Date de publication : 10 avril 2010

Pagination : 235-242

ISBN : 978-2-7535-1407-2

ISSN : $1960-1360$

Référence électronique

Jérôme Girard, Marguerite Munoz, Béatrice Cauuet, Mireille Polve, Sébastien Aries et Laurent Callegarin, « Mines d'argent du Montaigu (Hautes-Pyrénées, France)», ArcheoSciences [En ligne], 34 | 2010, mis en ligne le 11 avril 2013, consulté le 31 janvier 2022. URL : http://journals.openedition.org/ archeosciences/2792 ; DOI : https://doi.org/10.4000/archeosciences.2792 


\title{
Mines d'argent du Montaigu (Hautes-Pyrénées, France) : une filière aquitaine de l'argent? Étude isotopique du plomb
}

\author{
Silver mines on the massif of Montaigu (Hautes-Pyrénées, France): \\ An Aquitanian network for silver? Study on lead isotopes
}

\author{
Jérôme Girard*, Marguerite Munoz**, Béatrice Caunet*, Mireille Polve**, \\ Sébastien Aries ${ }^{* * *}$ et Laurent CALlegarin ${ }^{* * * *}$
}

\begin{abstract}
Résumé : Un nouveau programme thématique a mis en évidence trois sites d'extraction et de métallurgie argentifere de l'âge du Fer sur le massif du Montaigu (Hautes-Pyrénées) : la mine de Palouma sur le versant sud-ouest et la mine de Séras avec le site métallurgique proche d'Hourcade sur le versant nord-est. Une recherche de filiation a été engagée pour étudier la relation entre sites miniers et métallurgiques d'une part, et entre cette production d'argent et un monnayage aquitain contemporain d'autre part. La technique utilisée est le traçage isotopique du plomb. Les 12 échantillons analysés consistent en fragments de minerais, scories et monnaies, analysés par ICP-MS et selon un protocole adapté à notre démarche prospective. Les résultats montrent des signatures nettement différentes de celles des minéralisations du domaine pyrénéen déjà connues. Les signatures des minerais du Montaigu sont peu homogènes, y compris au sein d'un même gisement (Palouma), mais elles sont regroupées autour des signatures des scories du site d'Hourcade confortant l'hypothèse d'un traitement des minerais de Séras et Palouma sur le site métallurgique d'Hourcade. Enfin, si la composition isotopique d'une des monnaies est bien compatible avec nos gisements, l'autre indique que d'autres ressources sont impliquées. Cette étude souligne que le traçage des isotopes du plomb est pertinent pour la recherche d'anciennes provenances du métal à l'échelon local, mais aussi que cette technique nécessite d'être encadrée par une contextualisation archéologique, historique et géologique très précise.
\end{abstract}

\begin{abstract}
During a new thematic investigation three mining and metallurgical sites for silver from Iron Age have been discovered in the Montaigu massif (Hautes-Pyrénées): the Palouma mine on the southwestern mountain side and the Séras mine with the nearby metallurgic site of Hourcade on the north-eastern mountain side. A tracing study has been carried out in order to test the relationship: i) between the above cited sites and ii) between the silver produced in these sites and the contemporaneous Aquitain coinage. The lead isotopic tracing method has been used. The 12 samples, composed of ore, slag and coins fragments, have been analysed by ICP-MS with a protocol adapted to our prospective approach. Results evidence isotopic signatures drastically different from those of the Pyrenean ores already known. Isotopic compositions of ores from the Montaigu massif are not very homogeneous, even within the same deposit (Palouma) but they are close to slag values corroborating the hypothesis of Séras and Palouma ore processing at the Hourcade metallurgic site. Finally, one of the coins isotopic composition is compatible with the studied ores, while the second coin value indicates different sources are to be involved. This study highlights that lead isotopes tracing is appropriate for search of ancient metal networks at the local scale and the need of a precise archaeological, historical and geological context to frame this method.
\end{abstract}

Mots clé : Pyrénées, Âge du Fer, Argent, Isotopes du plomb, Mines.

Key words: Pyrénées, Iron Age, Silver, Lead isotopes, Mines.

* Université de Toulouse-Université du Mirail-CNRS, TRACES - 5 allée Antonio-Machado, 31058 Toulouse Cedex 9. (jerome.girard7@wanadoo.fr) (cauuetb@aol.com)

** Université de Toulouse-UPS-CNRS-IRD, LMTG-14av.E.-Belin, 31400 Toulouse-France. (mmunoz@lmtg.obs-mip.fr)(polve@lmtg.obs-mip.fr)

***Antellis - Impasse Palayré, 31100 Toulouse. (sebastien.aries@antellis.com)

*** Université de Pau et des Pays de l'Adour, GRA-JE 2245-Avenue du Doyen-Poplawski, BP 1160, 64013 Pau Cedex. (Laurent.callegarin@wanadoo.fr) 


\section{INTRODUCTION}

Dans le cadre d'un nouveau programme d'études des mines et métallurgies anciennes dans les Pyrénées centrales, des prospections thématiques menées depuis 2003 ont permis de constituer un corpus de sites archéologiques liés à l'exploitation des métaux non ferreux. Plusieurs gisements portent des témoignages d'exploitations préindustrielles, tels ceux de Largentière près de Marignac, des montagnes de Melles (Haute-Garonne) ou encore ceux des massifs du Cabaliros, du Nerbiou et du Montaigu (Haute-Pyrénées). Ce dernier, entre Bagnères-de-Bigorre et Lourdes, sur les premiers contreforts de la zone axiale de la chaîne des Pyrénées, a livré deux mines sur des gisements plombozincifères : la mine de Palouma (Gazost) et celle de Séras (Germs-sur-l'Oussouet). À cette dernière est associé le site métallurgique d'Hourcade, situé à proximité immédiate, qui présente un large épandage de scories argentifères en surface (fig. 1). Les recherches archéologiques anciennes et plus récentes faites sur ces trois sites ont permis de rassembler des indices chronologiques compris entre le $\mathrm{VI}^{\mathrm{e}}$ siècle avant notre ère et le $\mathrm{I}^{\text {er }}$ siècle de notre ère.

Dans le Massif du Montaigu, la possibilité de relier géographiquement une mine (Séras) et ses ateliers métallurgiques (Hourcade), ainsi que la présence d'une autre mine (Palouma), suffisamment proche pour être susceptible d'avoir alimenté ces mêmes ateliers, ont motivé la mise en

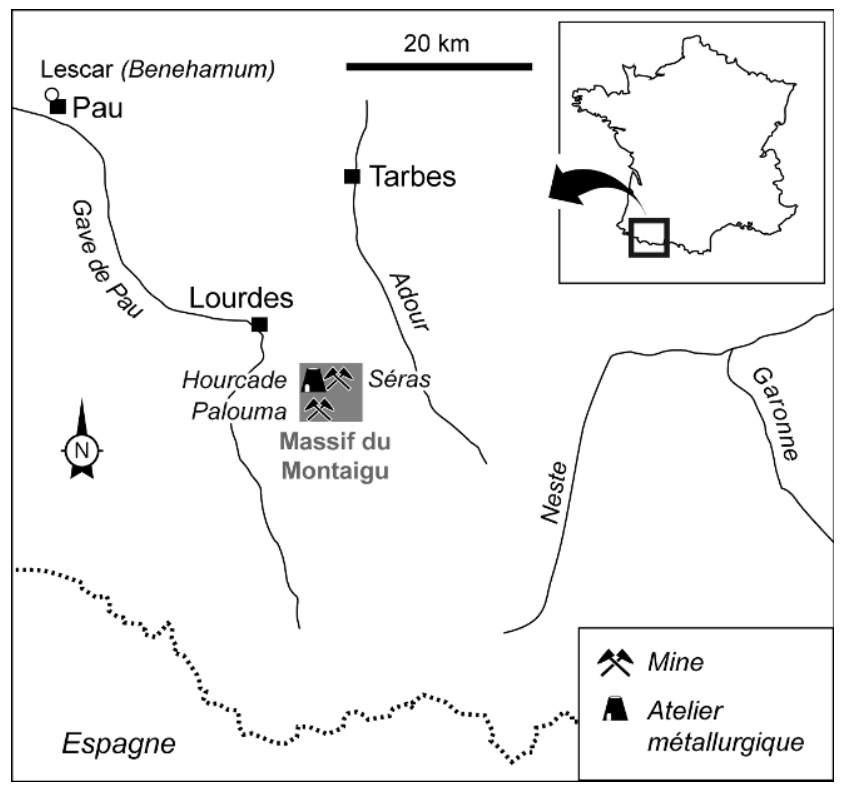

Figure 1 : Localisation du site de production métallique antique du Montaigu.

Figure 1: Localisation of the ancient metal production site of Montaigu. place d'une recherche de filiation à partir des minerais et scories prélevés in situ.

Par ailleurs, des recherches récentes sur les monnayages aquitains ont mis en évidence plusieurs centres émetteurs de monnaies d'argent préromaines. Ainsi, les oppida aquitains du Castéra à Labastide Montréjeau ou du Bilaà proche de la ville actuelle de Lescar située à $75 \mathrm{~km}$ du massif du Montaigu (Pyrénées-Atlantiques) et devenue Beneharnum, capitale des Benarni après la conquête romaine, paraissent être deux des centres émetteurs de monnaies d'argent frappées entre les $\mathrm{II}^{\mathrm{e}}$ et $\mathrm{I}^{\mathrm{er}}$ siècles avant notre ère (Callegarin $e t$ al., 2007).

Lobjectif de cette étude est la mise en relation par comparaison de la composition isotopique du plomb de trois des produits de la chaîne opératoire de production de l'argent, à savoir : des minerais argentifères prélevés dans les chantiers des mines datées de l'âge du Fer de Palouma et Séras, des scories argentifères du site métallurgique d'Hourcade attenant à la mine de Séras et deux des monnaies d'argent frappées à la même période et retrouvées dans le territoire des Benarni proche de la zone des lieux de production montagnards.

\section{ConteXte historique ET ARCHÉOLOGIQUE DE LA RECHERCHE}

Au second âge du Fer dans l'ensemble monétaire du Sudouest aquitain, les émissions monétaires "à protubérance " en argent, dites "Tarusates », se distribuent en deux séries, le type " Pomarez " et le type " Beyrie " (Callegarin, 2005). Cette dernière série présente au droit une protubérance circulaire latérale et striée de trois sillons à fort bourrelet central, produisant une image de "grain germé " (Callegarin et al., 2007). Le type "Beyrie " a une distribution très limitée, centrée autour de l'oppidum de Lescar près de Pau chez les Benarni, un des peuples aquitains (fig. 2). À l'inverse, le type "Pomarez " est diffusée plus largement dans les Landes, le Béarn et l'axe garonnais.

Les données métrologiques et les contextes archéologiques de ces deux monnayages permettent de dater les premières émissions dans le dernier quart du $\mathrm{II}^{\mathrm{e}}$ siècle av. J.-C., puis une circulation monétaire pendant tout le $\mathrm{I}^{\mathrm{er}}$ siècle av. J.-C. et jusqu'au tout début du $\mathrm{I}^{\text {er }}$ siècle de notre ère (époque Auguste-Tibère).

L’intérêt de la série monétaire du type Beyrie réside dans un espace de circulation étroit et situé au voisinage immédiat d'un massif montagneux, le Montaigu (au sud de Tarbes dans les Hautes-Pyrénées) où des recherches récentes ont mis en évidence une extraction minière d'argent. Cette activité 


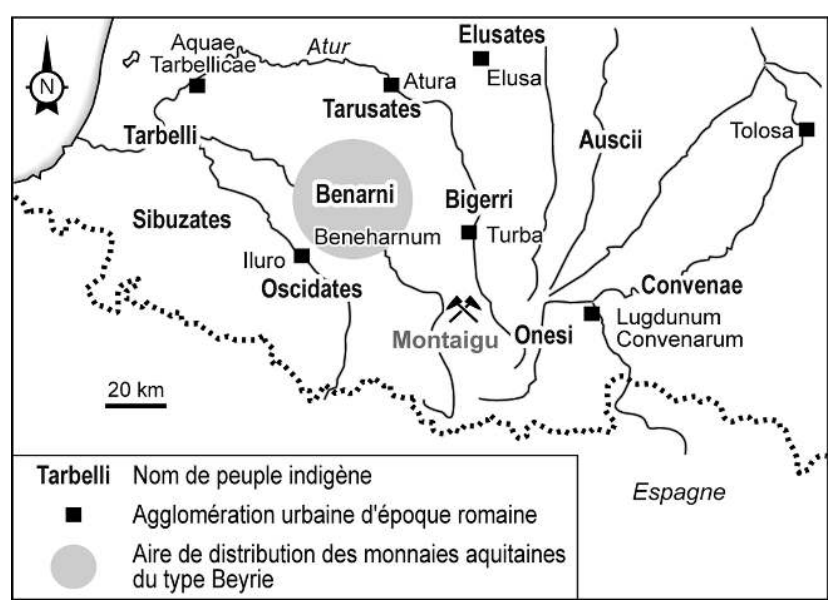

Figure 2: Contexte géopolitique des mines d'argent du Montaigu. Figure 2: Geopolitical context of the Montaigu silver mines.

a été datée de la fin du second âge du Fer dans les mines de Palouma et de Séras, ainsi que dans l'atelier métallurgique d'Hourcade proche des mines (Girard, 2003). La contemporanéité, au second âge du Fer, des deux activités dans un même espace économique séparé de quelque $70 \mathrm{~km}$ seulement, d'un côté la production d'argent et de l'autre la frappe de monnaies d'argent, autorise à tenter de restituer par la voie analytique et isotopique une des filières possibles de débouché de l'argent produit dans le massif du Montaigu, celle de l'approvisionnement en métal précieux d'un centre émetteur en monnaies d'argent à la fin du second âge du Fer dans le Sud-ouest aquitain (fig. 2).

Les caractéristiques physiques et historiques de ces monnaies (valeur forte, courte période d'émission et de circulation) les rendent peu susceptibles d'avoir été le produit d'un recyclage de métaux de diverses provenances. Ces arguments historiques, géographiques et archéologiques conduisent à considérer ces pièces d'argent comme le produit final d'un possible débouché aquitain de l'argent extrait du massif du Montaigu.

La recherche de la provenance des métaux utilisés dans les monnayages anciens (protohistoriques, antiques ou médiévaux) n'est pas nouvelle, notamment par l'analyse isotopique du plomb contenu dans le métal. L'argent et le plomb étant généralement associés dans le minerai, ce type d'analyse a commencé au début des années 1970 avec les travaux de Brill et Shields sur les premiers monnayages grecs en argent (Brill et Shields, 1972). Depuis cette époque les méthodes d'analyses ont évolué, les équipements se sont perfectionnés, permettant de travailler sur de faibles prélèvements concernant les objets. De même, à l'autre bout de la chaîne, la recherche des mines et donc l'analyse des minerais poten- tiellement exploités est devenue plus rigoureuse et en lien plus étroit avec les données de l'archéologie. En effet dans ce type d'approche et de confrontation entre minerai et objet métallique, le plus important réside dans la qualité et la représentativité des échantillons recueillis et analysés aux deux bouts de la chaîne. Aujourd'hui, on ne peut plus se contenter de prélèvements des minerais issus de contextes géologiques non documentés par l'archéologie. Il ne s'agit pas seulement de sélectionner par l'analyse des minerais naturels d'une composition proche de celle des objets étudiés, mais de confronter des minerais archéologiques, c'està-dire provenant de chantiers miniers datés, à des objets de la même époque, sur la base d'une problématique qui donne sens à l'étude (Tamas et al., 2009).

C'est en cela que la confrontation entre les minerais et les scories argentifêres, chrono- et géo- référencés, du massif du Montaigu et les monnayages du type Beyrie émis à la même époque dans le territoire des Benarni au second âge du Fer est pertinente. Le minerai exploité tant à Palouma qu'au Séras est de la galène argentifere riche en plomb. De même les scories argentifères recueillies sur l'atelier métallurgique d'Hourcade sont riches en plomb, l'analyse géochimique des isotopes du plomb s'est donc imposée pour confronter dans un même protocole analytique, des échantillons de minerais et de scories provenant des lieux de production du métal à deux monnaies d'argent du type Beyrie.

\section{Méthodes D’ANALyses}

Pour tester la validité de cette hypothèse, nous avons utilisé la technique du traçage isotopique. Les systèmes d'isotopes stables et radiogéniques sont utilisés en Sciences de la Terre et de l'Environnement comme traceurs de source et ont trouvé une application ces dernières années dans le domaine de l'archéologie, en particulier le système isotopique du plomb (Baron et al., 2006; Baron et al., 2009).

\section{Adéquation et limite des méthodes analytiques appliquées à l'archéologie}

La démarche est simple et consiste à comparer les compositions isotopiques d'un élément traceur pour établir ou exclure une filiation. Cette approche repose sur le principe de la conservation de la composition isotopique d'un élément chimique lourd lors des étapes de transformation du minerai à l'objet. La composition isotopique en plomb d'un gisement métallique est liée à l'âge de formation et n'évolue pas après la cristallisation des minéraux métalliques dans le gisement permettant de remonter à la source de production. 
Le plomb est l'élément traceur approprié dans l'archéologie des métaux non ferreux puisqu'il constitue le métal produit ou bien qu'il est associé à d'autres métaux exploités (Ag, $\mathrm{Cu} . .$.$) . De plus, il peut être analysé même en concentration$ très faible dans les objets d'argent, de cuivre, ou de bronze.

Des analyses isotopiques d'objets métalliques du début de l'âge du Bronze, trouvés dans le sud de l'Allemagne et la Bohème ont montré l'absence de relation avec les minerais de cette région exploités ultérieurement (Niederschlag, et al., 2003). De même, C. Domergue et son équipe ont étudié la circulation des lingots de plomb en Méditerranée et ont montré que les mines antiques du sud de l'Espagne constituaient une source importante de ce métal (Domergue et al. sous presse).

Cependant des limitations dans l'utilisation de cet outil existent, soit liées à la disponibilité des bases de données concernant les gisements, soit dues à l'hétérogénéité de signature dans un même gisement. Les limitations peuvent être liées aussi à l'homogénéité des signatures dans différents gisements géographiquement distants, soit liées à la possibilité de mélanges de minerais dans un site métallurgique ou encore d'ajouts lors du processus métallurgique et finalement au recyclage du métal. Ainsi, Attanasio et al. (2001) ont analysé des monnaies puniques en bronze du iII siècle avant J.-C. trouvées à Tharros (Sardaigne occidentale) et concluent sur la possibilité d'un intense recyclage du métal. Les recherches de Boni et al. (2000) sur des conduites en plomb de Pompei concluent aussi à la difficulté de remonter au minerai à partir d'objets finis.
Dans le cadre de cette étude préliminaire sur un débouché antique de l'argent, un protocole analytique allégé a été utilisé pour tester la possibilité de filiation entre les différents sites de production du Montaigu (les minerais de deux mines et les scories d'un site métallurgique datés et contemporains et à titre de comparaison le minerai de la mine moderne de Lia, proche de ces sites) et des échantillons issus d'un corpus monétaire préromain (deux pièces d'argent) dont le contexte historique et archéologique en fait un possible débouché de l'argent produit.

\section{Échantillonnage et prélèvements sur échantillons}

Au sein de l'ensemble étudié, les sites sélectionnés Palouma, Séras, Lia et Hourcade sont regroupés au sein d'une unité géographique (massif du Montaigu), et/ou d'une unité chronologique ( $\mathrm{II}^{\mathrm{e}}$ siècle av. n. è./ $\mathrm{I}^{\mathrm{er}}$ siècle de n. è.). Ces sites sont donc susceptibles d'avoir fonctionné " en réseau ». Si la mine de Séras est à l'évidence celle qui a fourni les ateliers d'Hourcade (à quelques centaines de mètres), la mine de Palouma, située à $4 \mathrm{~km}$, a aussi pu être pourvoyeuse de minerais. Dans la perspective de confronter nos données isotopiques à celles d'autres minerais argentiferes pyrénéens et proches des sites miniers archéologiques étudiés, le minerai de la mine moderne de Lia (partie sud du massif du Montaigu) a aussi été analysé. La mesure des signatures isotopiques du plomb a été effectuée sur plusieurs types de matériaux plombifères (tabl. 1).

\begin{tabular}{|c|c|c|c|c|c|c|c|c|}
\hline Site & Échantillon & Phase analysée & $\frac{\mathrm{Pb}^{207}}{\mathrm{~Pb}^{206}}$ & $2 \sigma$ & $\frac{\mathrm{Pb}^{208}}{\mathrm{~Pb}^{206}}$ & $2 \sigma$ & $\frac{\mathrm{Pb}^{208}}{\mathrm{~Pb}^{207}}$ & $2 \sigma$ \\
\hline Séras & minerai & SER 1/oxyde Mn-Pb & 0,8446 & 0,0043 & 2,0732 & 0,0090 & 2,4517 & 0,0099 \\
\hline \multirow[t]{3}{*}{ Palouma } & minerai & M1B/galène & 0,8357 & 0,0041 & 2,0653 & 0,0085 & 2,4683 & 0,0096 \\
\hline & minerai & M2C/galène & 0,8360 & 0,0043 & 2,0567 & 0,0088 & 2,4569 & 0,0095 \\
\hline & minerai & M3C/galène & 0,8458 & 0,0044 & 2,0918 & 0,0088 & 2,4596 & 0,0096 \\
\hline Lia & minerai & LIA 1/galène & 0,8575 & 0,0043 & 2,0927 & 0,0092 & 2,4370 & 0,0093 \\
\hline \multirow[t]{5}{*}{ Hourcade } & scorie & Hourc4/verre & 0,8423 & 0,0042 & 2,0731 & 0,0092 & 2,4570 & 0,0098 \\
\hline & scorie & Hourc10/ plomb & 0,8339 & 0,0043 & 2,0565 & 0,0090 & 2,4644 & 0,0104 \\
\hline & scorie & Hourc10/ verre & 0,8362 & 0,0043 & 2,0688 & 0,0092 & 2,4704 & 0,0098 \\
\hline & scorie & Hourc9/ plomb & 0,8439 & 0,0042 & 2,0697 & 0,0088 & 2,4498 & 0,0095 \\
\hline & scorie & Hourc9 / verre & 0,8441 & 0,0040 & 2,0684 & 0,0089 & 2,4473 & 0,0097 \\
\hline \multirow[t]{7}{*}{ Labastide } & monnaie & LAB 2/argent & 0,8414 & 0,0042 & 2,0704 & 0,0087 & 2,4584 & 0,0095 \\
\hline & monnaie & LAB 3/argent & 0,8267 & 0,0041 & 2,0357 & 0,0088 & 2,4613 & 0,0096 \\
\hline & NBS SRM 981 & Matériel de référence & 0,9201 & 0,0038 & 2,2310 & 0,0091 & 2,4155 & 0,0093 \\
\hline & NBS SRM 981 & Matériel de référence & 0,9252 & 0,0037 & 2,2309 & 0,0093 & 2,4032 & 0,0091 \\
\hline & NBS SRM 981 & Matériel de référence & 0,9216 & 0,0038 & 2,2332 & 0,0092 & 2,4140 & 0,0090 \\
\hline & NBS SRM 981 & Matériel de référence & 0,9210 & 0,0039 & 2,2157 & 0,0088 & 2,3975 & 0,0092 \\
\hline & NBS SRM 981 & Valeurs certifiées & 0,9146 & 0,0007 & 2,1677 & 0,0016 & 2,3704 & 0,0010 \\
\hline
\end{tabular}

Tableau 1 : Composition isotopique des échantillons analysés et écart type ( $\sigma)$.

Table 1: Isotopic composition of analysed samples and standard deviation ( $\sigma)$. 
Pour les minerais de Palouma et de Lia, les prélèvements ont été effectués dans la galène (sulfure de plomb, $\mathrm{PbS}$ ) qui constitue le minéral porteur de l'argent ou qui lui est associé. En ce qui concerne le minerai de Séras, le prélèvement a été effectué dans des nodules d'oxyde de manganèse qui est la seule phase plombifère identifiée, par microsonde électronique, dans ce gisement.

Dans les scories de Hourcade, le plomb métal s'individualise en billes parfois plurimillimétriques dans la matrice vitreuse (fig. 3) et les deux phases ont été analysées.

Deux monnaies du type "Beyrie » trouvées à LabastideMontréjeau, près de Lescar, ont pu être intégrées aux analyses : Labastide 2 (Ag 98,4 \%, Cu 0,9\% Pb 0,4\%) et Labastide 3 (Ag 96,6\%, Cu 2,5\%, Pb 0,9\%). Sous une pellicule d'oxydation, le cœur métallique est bien conservé. Des prélèvements de 10 à $20 \mathrm{mg}$ ont été réalisés manuellement sur sections polies pour les minerais et les scories et directement sur les pièces de monnaies après avoir ôté la pellicule d'oxydation.

\section{Protocole analytique}

La méthode d'analyse classiquement utilisée est le TIMS qui donne des résultats reproductibles et précis. Dans le cadre de cette étude préliminaire notre préférence s'est portée sur un protocole simplifié. Elles ont été effectuées par ICP-MS (Perkin Elmer, Elan 6000), en solution après digestion acide avec $\mathrm{HNO}_{3}$ et $\mathrm{HF}$ bi-distillés et dilution, sans étape de purification sur résine. Baker et al. (2006) ont montré que la présence de matrice dans le cas d'une dis-

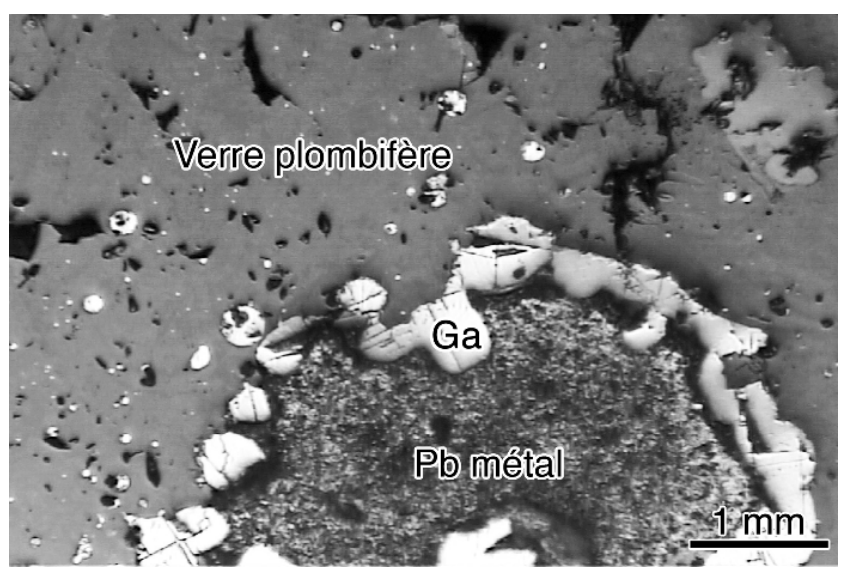

Figure 3 : Microphotographie de scorie en lumière réfléchie : nodule de plomb métal, entouré de galène $(\mathrm{Ga})$ au sein de la matrice de verre plombifere.

Figure 3: Photomicrograph of slag sample under reflected light: metallic lead nodule, rounded by galena $(G a)$ within the lead bearing glassy matrix. solution totale sans purification du plomb n'induisait pas d'interférences isobariques ni de variations du biais de masse significatifs lors de l'analyse par ICP-MS. La précision est plus faible qu'avec le TIMS mais elle est suffisante. Ce protocole, plus rapide et moins cher est donc adapté pour des analyses prospectives. Le standard NBS 981 dilué à $30 \mathrm{ppb}$ a été analysé encadrant 3 ou 4 échantillons pour corriger les échantillons par la technique du bracketing décrite par Maréchal et al. (1999).

\section{RÉSULTATS ET DISCUSSION}

Les compositions isotopiques des minerais et des scories, ainsi que celles des monnaies (tab. 1) ont été reportées dans un diagramme $\mathrm{Pb}^{208} / \mathrm{Pb}^{206}$ versus $\mathrm{Pb}^{207} / \mathrm{Pb}^{206}$ (fig. 4). Elles ont ensuite été comparées à la signature isotopique des autres sites pyrénéens connus (Marcoux et Moëlo, 1991; Marcoux et al., 1991; Velasco et al., 1996).

Les résultats montrent que, compte tenu de la barre d'erreur sur les mesures, on peut considérer qu'une majorité d'échantillons présente une signature isotopique en plomb très voisine. Cet ensemble est constitué de l'oxyde de manganèse plombifère de Séras, de deux des trois galènes de Palouma, des scories et de la pièce d'argent Labastide 2. En revanche, trois échantillons s'écartent sensiblement de cet ensemble, la galène de Lia, d'un des échantillons de Palouma et de la pièce de monnaie Labastide 3.

Ainsi, on peut constater que les minéralisations du massif du Montaigu ne présentent pas une signature isotopique homogène, même à l'échelle du gisement de Palouma. La minéralisation de Lia et une partie de la minéralisation de Palouma seraient liées à un contexte métallogénique distinct, mais plus proche de celui des gisements pyrénéens de la base de données existante. L'hétérogénéité des signatures isotopiques au sein d'une même mine (Palouma) complexifie la démarche de filiation en même temps qu'elle est porteuse d'une discrimination plus précise.

Les signatures des scories du site d'Hourcade regroupées autour des valeurs des minerais de Séras et de Palouma confortent l'hypothèse d'un traitement de ces deux minerais sur le site métallurgique d'Hourcade. La différence de signature avec le minerai de Lia exclut ce dernier comme source du plomb d'Hourcade, ce que corrobore l'archéologie : c'est la seule minéralisation de l'ensemble considéré qui ne présente aucun vestige d'exploitation préindustrielle.

Concernant les monnaies, la signature isotopique de la pièce d'argent Labastide 2, intermédiaire entre les valeurs obtenues pour les scories d'Hourcade, est compatible avec une source locale. Ceci n'exclut toutefois pas une contribu- 


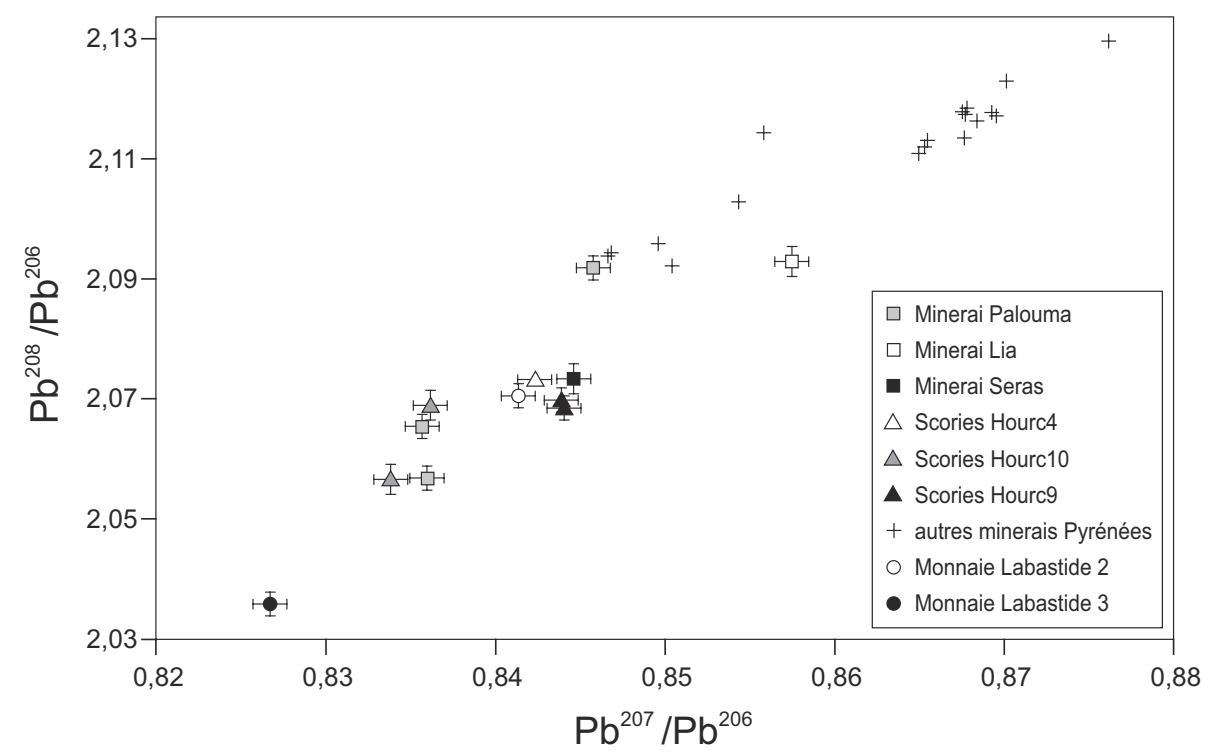

Figure 4: Composition isotopique (avec barres des écarts type) des minerais de Palouma, Lia et Séras, des scories de Hourcade et des pièces d'argent de Labastide. Positionnement d'autres minéralisations pyrénéennes d'après la bibliographie.

Figure 4: Isotopic composition (with standard deviation bars) of ore samples from Palouma, Lia et Séras, slag samples from Hourcade and of silver coins from Labastide. Plotting of other pyrenean mineralisation compositions from literature. tion extérieure. La signature isotopique de la pièce de monnaie Labastide 3, significativement différente, implique que l'argent ne proviendrait pas des gisements étudiés à ce jour sur le massif du Montaigu. Il ne provient pas non plus des autres gisements pyrénéens de la bibliographie. Ce résultat impose de rechercher d'autres sources de minerais, locales ou plus lointaines. Enfin, il faut garder à l'esprit que le recyclage des métaux peut être un élément fortement perturbateur dans ces études de filiation.

\section{Conclusion}

En dépit de leur nature prospective, les données isotopiques obtenues mettent en évidence le potentiel d'une étude des isotopes du plomb au service de l'histoire. Elles permettent ici de tenter de restituer les filières de diffusion et d'utilisation de l'argent pyrénéen à l'âge du Fer, entre un centre minier et métallurgique montagnard (district du massif du Montaigu, Hautes-Pyrénées) et le centre monétaire émetteur de Lescar (près de Pau), oppidum " capitale " supposée des Benarni, peuple aquitain. Le questionnement principal, celui de la filière économique, trouve par la géochimie des éléments de réponse grâce à l'adéquation entre les signatures isotopiques de certains minerais exploités et traités par les Aquitains et au moins une des deux monnaies Benarni analysées.

Ce travail met en évidence la possibilité d'une contribution des mines du Montaigu au monnayage aquitain sud-occidental de type Beyrie, mais aussi la diversité d'ap- provisionnement des ateliers monétaires et/ou l'existence d'autres gisements sources dans la région.

Au sein de l'ancienne zone de production d'argent du Montaigu, la géochimie isotopique montre que des relations entre sites distants sont possibles (traitement du minerai de Palouma aux ateliers métallurgiques d'Hourcade). Elle indique donc de nouvelles pistes de recherches pour l'archéologie. Au chapitre du débouché, l'hypothèse formulée, grâce à des indices historiques, d'une filière "MontaiguLescar " reste encore à consolider, notamment en analysant un plus grand nombre de monnaies de type "Beyrie " et en poursuivant le programme de prospection minière et métallurgique pour vérifier plus largement les sources pyrénéennes de l'argent. En effet, la dissemblance des signatures des monnaies implique des sources encore inconnues.

Le constat fait sur la diversité des signatures de minerais à Palouma doit être précisé. On ne peut donc plus parler de la signature d'une mine ancienne, mais de son éventail de signatures. Il montre qu'une connaissance géologique fine des anciennes mines devient une obligation dans toute étude de filiation, et qu'il faut constituer une base de données exhaustive des gisements et mines pyrénéennes.

\section{Remerciements}

Notre gratitude va tout particulièrement à Christiane Hester, dessinatrice au Laboratoire LMTG (UMR 5563), pour la qualité de ses figures, ainsi qu'au relecteur pour ses remarques constructives. 


\section{BibliograPHIE}

Attanasio, D., Bultrini, G. et Ingo, G. M., 2001. The possibility of provenancing a series of bronze Punic coins found at Tharros, (western Sardinia) using the literature lead isotope database, Archaeometry, 43, (4), p. 529-547.

BaKer, J., Stos, S. et Waight, T., 2006. Lead isotope analysis of archaeological metals by multiple-collector inductively coupled plasma mass spectrometry, Archaeometry 48, (1), p. 45-56.

Baron, S., Carignan, J., Laurent, S. et Ploquin, A., 2006. Medieval lead making on Mont-Lozère Massif (CévennesFrance): Tracing ore sources using $\mathrm{Pb}$ isotopes, Applied Geochemistry, 21, p. 241-252.

Baron, S., Le-Carlier, C., Carignan, J. et Ploquin, A., 2009. Archaeological reconstruction of medieval lead production: implications for ancient metal provenance studies and paleopollution tracing by $\mathrm{Pb}$ isotopes, Applied Geochemistry, 24, p. 2093-2101.

Boni, M., Di Maio, G., Frei, R. et Villa, I. M., 2000. Lead isotopic evidence for a mixed provenance for Roman water pipes from Pompeii. Archaeometry, 42, p. 201-208.

Brill, R. H. et SHIElds, W. R., 1972. Lead isotopes in Ancient coins. Methods of chemical and metallurgical investigations of Ancient coinage, Hall \& Metcalf (ed.), Londres, p. 279-303.

Callegarin, L., 2005. Le monnayage dit tarusate (sud-ouest de la Gaule) : révisions et perspectives XIII congreso internacional de numismatica (Madrid 2003), Madrid, p. 427-440.

Callegarin, L., collaboration Guimon, M.-F., Castetbon, A. et GIRARD, J., 2007. L'ensemble monétaire « aquitain sud-occidental " au second âge du Fer : une première approche. In Les âges du Fer dans le Sud-ouest de la France, XXVIII ${ }^{\mathrm{e}}$ colloque de l'AFEAF (Toulouse, 20-23 mai 2004), Ed. Vaginay M. et IzacImbert L., Aquitania Supplément 14/1, Bordeaux, p. 209-226.

Domergue, C., Quarati, P. et Trincherini, P. R. (sous presse). Les méthodes de l'archéométrie et des sciences de l'Antiquité appliquées aux lingots de plomb de l'épave de Comacchio : le problème de leur origine et de leur signification, in Orejas, A., Rico, C. et Sánchez Palencia, J. (dir.), Mineria antigua : estudios regionales y temas de investigacion actual, Colloque International 28-29 novembre 2005, Casa de Velazquez, Madrid.

Girard, J., 2003. Plomb et argent dans les Pyrénées centrales. Prospection thématique, Bilan Scientifique de la Région MidiPyrénées, 2003, p. 183.

Maréchal, C., Télouk, P. et Albarède, F., 1999. Precise analysis of copper and zinc isotopic compositions by plasma-source mass spectrometry, Chemical Geology, 156, p. 251-273.

MarCoux, E. et MoËLo, Y., 1991. Lead isotope geochemistry and paragenetic study of inheritance phenomena in metallogenesis: examples from base metal sulfide deposits in France, Economic Geology, 86, p. 106-120.

Marcoux, E., Joubert, M. et Lescuyer, J.-L., 1991. Origine des minéralisations stratiformes de la bordure du Canigou (Pyrénées-Orientales, France); apport de la géochimie isotopique du plomb, Comptes Rendus de l'Académie des Sciences, 312, p. 281-287.

Niederschlag, E., Pernicka, E., Seifert, T. H., Bartelheim, M., 2003. The determination of lead isotope ratios by multiple multicollector ICP-MS: a case study of early Bronze Age artefacts and their possible relation with ore deposits of the Erzgebirge. Archeometry, 45, p. 61-100.

Tamas, C.G., Baron, S., Cauuet, B., 2009. Minéralogie et signature isotopique du plomb des minerais auro-argentifères exploités durant l'époque romaine à Alburnus Maior (Roşia Montană, Roumanie), Actes du Colloque international AURUM Authentification and Analysis of Gold Work, Paris, 11-13 mai 2009, Archeosciences, p. 83-89.

Velasco, F., Pesquera, A., Herrero, J. M., 1996. Lead-Isotope Study of $\mathrm{Zn}-\mathrm{Pb}$ Ore Deposits Associated with the BasqueCantabrian Basin and Paleozoic Basement, Northern Spain. Mineralium Deposita, 31, p. 84-92. 
